\title{
Systematic Analysis of the Whole-Body Tissue Distribution and Fatty Acid Compositions of Membrane Lipids in CD1 and NMRI Mice and Wistar Rats
}

\author{
Lina Xu, ${ }^{1,2}$ Maximilian V. Schmitt, ${ }^{3}$ Huabin Ruan, ${ }^{1,2}$ Yupei Jiao, ${ }^{1,2}$ Xueying Wang, ${ }^{1,2}$ \\ Yusong Wang, ${ }^{1,2}$ Tao Yang, ${ }^{1,2}$ Philip Lienau, ${ }^{3}$ Andreas Reichel, ${ }^{3}$ and Xiaohui Liu $\mathbb{D}^{1,2}$ \\ ${ }^{1}$ School of Life Sciences, Tsinghua University, Beijing, China \\ ${ }^{2}$ National Protein Science Facility (Beijing), Tsinghua University, Beijing, China \\ ${ }^{3} D M P K$, Pharma ReD, Bayer AG, Berlin, Germany
}

Correspondence should be addressed to Xiaohui Liu; xiaohui2013@mail.tsinghua.edu.cn

Received 17 August 2020; Revised 12 November 2020; Accepted 19 November 2020; Published 30 November 2020

Academic Editor: Barbara Bojko Copyright (C) 2020 Lina Xu et al. This is an open access article distributed under the Creative Commons Attribution License, which
permits unrestricted use, distribution, and reproduction in any medium, provided the original work is properly cited.

Understanding the tissue distribution of phospholipids and glycerolipids in animal models enables promoting the pharmacokinetic study of drugs and related PK predictions. The measurement of lipid compositions in animal models, usually mice and rats, without a standardized approach hindered the accuracy of PBPK investigation. In this work, high resolution mass spectrometry was applied to profile the tissue distribution of phospholipids and glycerolipids in 12 organs/tissues of mice and rats. Using this method, not only the amounts of phospholipids and glycerolipids in each organ/tissue but also the fatty acid compositions were acquired. In order to explore the interspecies specificity of lipid distribution in different organs/tissues, three animal species including CD1 mice, NMRI mice, and Wister rats were used in this systematic study. Globally, more organ specificity was observed. It was found that the brain is the organ containing the most abundant phosphatidylserine lipids (PSs) in all three animal models, leading to brain tissues having the most concentrated acidic phospholipids. Diverse fatty acid compositions in each lipid class were clearly revealed. Certain tissues/organs also had a specific selection of unique fatty acid compositions, for example, unreferenced $\mathrm{FA}(18: 2)$ in the brain. It turned out that the access of free fatty acids affects the incorporation of acyl chain in phospholipids and glycerolipids. In the analysis, ether lipids were also profiled with the observation of dominant ePEs in brain tissues. However, little interspecies difference was found for fatty acid constituents and tissues distribution of phospholipids and glycerolipids.

\section{Introduction}

Phospholipids and glycerolipids are major lipid constituents of cell membranes. They are also involved in many cellular processes in health and disease. Various diseases have been reported to correlate with lipid metabolism [1-5] and several studies revealed changes in lipid regulation in animal and human tissues for different diseases [6-10]. The primary function of the highly abundant phospholipids and glycerolipids though is to support the structure of the cellular membrane. The lipid composition of cell membranes and tissues plays an important role in determining the pharmacokinetics (PK) of drugs, in particular their tissue partitioning which to a large extent is controlled by the lipid components in the body tissues [11-15]. There is great interest in accurate a priori predictions of drug-tissue partition coefficients based on the tissue composition in human or preclinical animal species ${ }^{15}$. Physiologically based PK (PBPK) models, therefore, need to account for species differences in lipid constituents that determine drug-tissue distribution. Although previous reports suggest the existence of species differences in the lipid tissue composition [16], lipid constituents in various animal strains have mostly been investigated individually in different studies using 
different protocols [17-19]. However, measurements using different methods and techniques may introduce a significant amount of bias into interspecies comparison and their corresponding PK predictions and different tissue dissection and storage conditions may also lead to inaccuracies of lipid investigation. Therefore, there is a need for a more systematic analysis of animal species and strain differences of tissue lipid constituents using the same analytical approach.

Mass spectrometry (MS) is a powerful tool for comprehensive lipidomic analysis including accurate molecular identification and quantitation. Multiple analytical platforms with MS have been developed. Shotgun lipidomics based on direct infusion was first introduced for complex lipid analysis using distinctive neutral loss scans [20-24]. Coupling of liquid chromatography (LC) powers and simplifies large-scale analysis of MS for complicated biological systems. Targeted lipidomics using LC-MS/MS is an alternative method for lipid analysis [25-27]. Selected reaction monitoring (SRM) is applied for data acquisition, supplying high sensitivity and wide linearity. Recently, high resolution MS has become more popular for comprehensive lipid analysis [28-31]. Data-dependent acquisition (DDA) enables confident lipid identification based on MS/MS assignment. The presence of abundant MS/MS spectral libraries, for example, LipidBlast, LIPID MAPS, or LipidSearch, allows the comprehensive analysis of lipidome in cells and animals [32-35].

In this work, phospholipids including phosphatidylcholine (PC), phosphatidylethanolamine (PE), phosphatidylinositol (PI), phosphatidylserine (PS), phosphatidylglycerol (PG), cardiolipin (CL), and lysophospholipids (LPLs) and glycerolipids including diglyceride (DG) and triglyceride (TG) in 12 organs/tissues of Wistar rats and CD1 and NMRI mice were profiled using Orbitrap based high resolution mass spectrometry. Lipid identification using DDA and MS-based quantitation was applied. Specific strategies for false positive elimination were used to improve the confidence of lipid analysis. Using this method, the fatty acid constituents of phospholipids and glycerolipids could be resolved. This allows the comparison of lipid composition in the animal organs of species and strains. Ether lipids including plasmanyl- and plasmenyl-phospholipids were also analyzed to explore the animal organ or species dependency of acyl chain components.

\section{Materials and Methods}

2.1. Sample Collection of Rats and Mice. All animal experiments were conducted in accordance with the German animal welfare laws and approved by local authorities. Animals were kept on a diet based on rodent's ssniff ${ }^{\mathrm{TM}}$ pellets "Ratte/Maus-Haltung" (ssniff-Spezialdiäten $\mathrm{GmbH}$, Soest, Germany) and water ad libitum. Tissue samples of brain, heart, liver, kidney, spleen, stomach, lung, muscle, small intestine, large intestine, skin, and fat were collected from individual male Wistar rats (HsdRCCHan:Wistar, Envigo, NL; weight range: 256-302 g), female NMRI mice (Crl:NMRI BR, Charles River, Germany; weight range: 28-30 g), and female CD1 mice (Crl:CD1(Icr), Charles
River, Germany; weight range: $27-31 \mathrm{~g}$ ) with nine replicates, respectively. Solid tissues were excised, briefly washed with isotonic $\mathrm{NaCl}$ solution to wash off blood residues. The stomach and the intestines were additionally cleared of their content with $\mathrm{NaCl}$ washing solution. The whole organs were collected, except for skin $\left(4 \mathrm{~cm}^{2}\right.$, belly), fat ( $5 \mathrm{~g}$, abdominal fat), and muscles ( $5 \mathrm{~g}$, belly/leg). All samples were immediately shock frozen in liquid nitrogen after preparation.

\subsection{Lipid Extraction from 12 Organs/Tissues of Wistar Rat and} CD1 and NMRI Mice. Lipids were extracted as previously described [36]. Briefly, $50 \mathrm{mg}$ wet tissue samples were homogenized in $500 \mu \mathrm{L}$ of chloroform/methanol $(v: v=2: 1)$ with an internal standard solution. Aqueous was subsequently added. Solutions were vortexed for 1-2 min and prepared for extraction with three replicates. Then, samples were centrifuged for 5 mins at $1000 \mathrm{rpm}$ for phase separation. The lower organic layer was transferred using a glass syringe and dried under nitrogen. Lipid samples were stored in $-80^{\circ} \mathrm{C}$ dry pellets. During lipid extraction, $5 \mathrm{~mL}$ glass tubes were used in order to avoid contamination.

2.3. Lipidomic Analysis Using LC-MS/MS. Reverse phase chromatography was selected for LC separation using CORTECS C18 Column $(2.1 \times 100 \mathrm{~mm}$, Waters $)$. Mobile phase A was made by mixing $400 \mathrm{~mL}$ of HPLC-grade water containing $0.77 \mathrm{~g}$ of ammonium acetate with $600 \mathrm{~mL}$ of HPLC-grade acetonitrile ( $\mathrm{pH} \sim 7)$. Mobile phase B contained $10 \% \mathrm{ACN}$ and $90 \%$ IPA (v/v). Data was acquired using Q Exactive Orbitrap mass spectrometer (Thermo, CA) coupled with UHPLC system Ultimate 3000 (Thermo, CA). Datadependent acquisition (DDA) was performed with 70,000 MS resolution and 17,500 MS/MS resolution. MS/MS spectra were acquired from the top 10 most intense precursors. Stepped NCE (normalized collision energy) of 15, 25,35 was applied for fragmentation.

2.4. Identification of Phospholipids and Glycerolipids. Lipids were identified using LipidSearch software v4.1.16 (Thermo, CA). LipidSearch v4.1.16 has 1,500,000 fragment ions of 71 subspecies in the database. It allows lipid identification based on MS/MS match [23] and ID-based quantitation was performed. Mass tolerance of $8 \mathrm{ppm}$ and 15 ppm was set for precursor and fragment, respectively. The displayed $\mathrm{m}$-score threshold was selected as 5 and grades $\mathrm{A}$, $\mathrm{B}, \mathrm{C}, \mathrm{D}$ were all used for ID quality filter. Adducts of $+\mathrm{H}$, $+\mathrm{NH} 4$ were used for positive mode search and $-\mathrm{H}$, $+\mathrm{CH} 3 \mathrm{COO}^{-}$were chosen for negative mode. Thresholds of $\mathrm{m}$-core and chromatographic peak shapes were applied to eliminate false positives.

2.5. Quantitation of Phospholipids and Glycerolipids. Stock solutions of $10 \mathrm{mg} / \mathrm{mL}$ PC $(18: 0 / 18: 0)$, PE $(18: 0 / 18$ : $0)$, PI $(16: 0 / 16: 0)$, PS $(16: 0 / 16: 0)$, PG $(18: 0 / 18: 0)$, CL $(18: 1 / 18: 1 / 18: 1 / 18: 1)$, DG $(16: 0 / 18: 0)$, and TG $(16: 0 / 16$ : $0 / 18: 0)$ were prepared. Then, a series of calibration solutions, with concentrations of from $1 \mathrm{ng} / \mathrm{mL}$ up to $200 \mu \mathrm{g} / \mathrm{mL}$, 


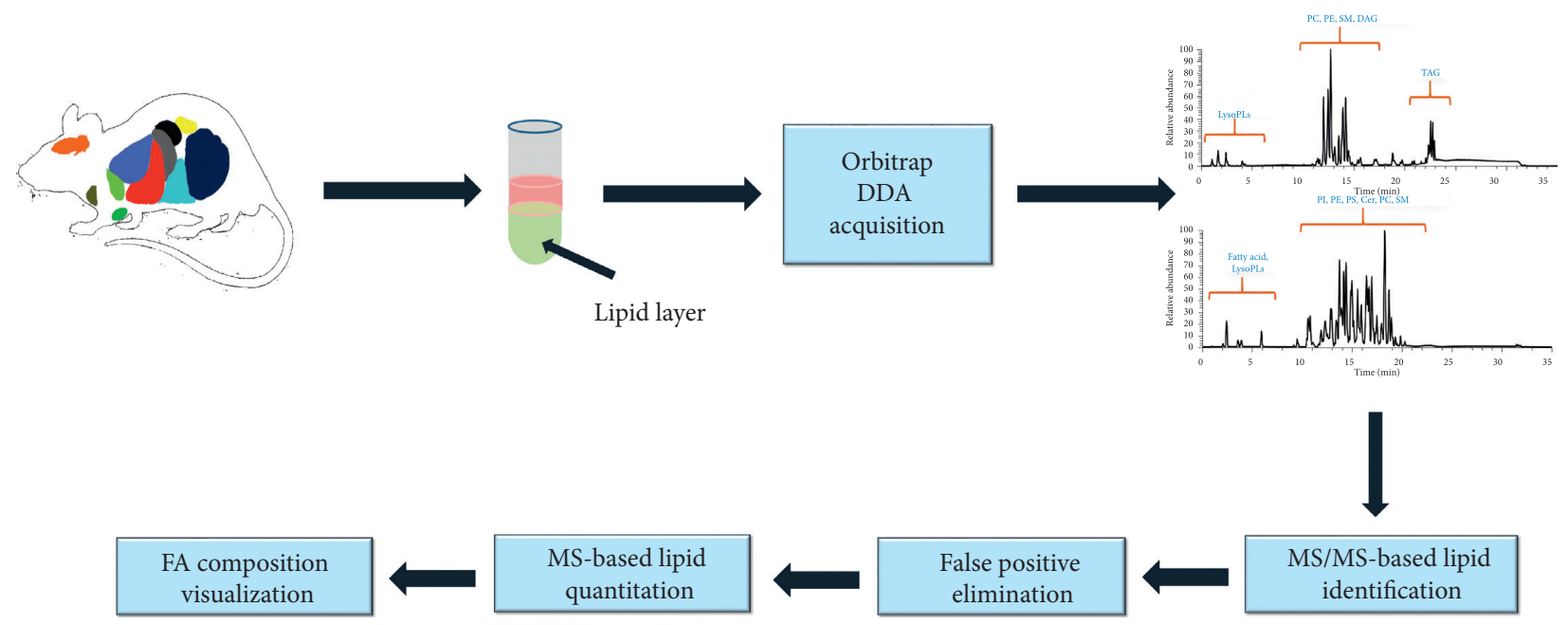

FIGURE 1: Schematic showing the workflow to perform lipidomics analysis for tissues/organs.

were diluted using $\mathrm{CH} 2 \mathrm{Cl} 2: \mathrm{MeOH}=2: 1$. Internal standard solution containing PC $(14: 0 / 14: 0), \mathrm{PE}(14: 0 / 14: 0), \mathrm{PI}(8$ : $0 / 8: 0)$ PS $(14: 0 / 14: 0)$, PG $(14: 0 / 14: 0)$, CL $(14: 0 / 14: 0 /$ $14: 0 / 14: 0)$, DG $(14: 0 / 14: 0)$, and TG $(16: 0 / 16: 0 / 18: 0)$ D7 were made. Equal volumes of calibration solution and internal standard solution were mixed. Injection volume of $1 \mu \mathrm{L}$ was used for the analysis.

2.6. Analysis of Glycerophospholipids and Glycerolipids in Mouse Brain Tissues. C57BL/6 mice at the age of 12 and 96 weeks were dissected and lipids in frozen brain tissues were extracted according to the protocols in section 2.2. Five replicates were used in the analysis. Glycerophospholipids were analyzed in negative ion mode using UHPLC-Q Exactive Orbitrap MS whereas DG and TG lipid molecules were analyzed in positive ion mode. The acyl chain component analysis was performed for each lipid class based on the abundance of lipid molecules. The abundance of lipid molecule was considered to contribute equally to each fatty acid component. Therefore, the percentage of specific acyl chain components in a certain lipid class was calculated using the total abundance of lipids containing this component divided by the total abundance of all lipid molecules in this class.

\section{Results and Discussion}

3.1. Analysis of Phospholipids and Glycerolipids in Mice and Rats. In this analysis, data-dependent MS/MS acquisition (DDA) was applied for lipid identification with MS-based quantitation as shown in Figure 1. LipidSearch, which contains millions of predicted fragment ion information was applied to assign lipid molecules based on MS/MS fragments. However, due to the extraordinary presence of fake molecular identification using LipidSearch, the strategies to remove unreliable assignments were utilized to reduce false positive results [37]. Briefly, the chromatographic behavior and MS/MS match were evaluated for each lipid species. The molecules with poor peak shapes or low confidence of MS/
MS matching score (m-score) were eliminated as false positives. However, the threshold of peak shape or m-score was unique for each lipid class depending on the data quality. Moreover, the signals from in-source decay or polymerization also had to be excluded as artificial ones, for example, dimethylated $\mathrm{PE}(\mathrm{dMePE})$ or fatty acid ester of hydroxyl fatty acid (FAHFA) [37].

For lipid quantitation, exogenous internal standards including PC(14:0/14:0), PE $(14: 0 / 14: 0)$, PS (14:0/14:0), PG (14:0/14:0), CL (14:0/14:0/14:0/14:0), DG (14:0/14: $0)$, and TG $(16: 0 / 16: 0 / 18: 0)-D 7$ were applied for quantification of each lipid class with PG $(14: 0 / 14: 0)$ also used as an internal standard of phosphatidylinositol (PI). PC (18:0/ $18: 0)$, PE (18:0/18:0), PI (16:0/16:0), PS (16:0/16:0), PG $(18: 0 / 18: 0)$, CL $(18: 1 / 18: 1 / 18: 1 / 18: 1)$, DG $(16: 0 / 18: 0)$, and TG $(16: 0 / 16: 0 / 18: 0)$ were selected as representative molecules for quantitation. Generally, glycerolipids generate the featured fragment of the head group or neutral loss in positive mode and fatty acid fragments in ion negative mode, whereas triglyceride (TG) and diglycerides (DG) were observed to have a unique neutral loss of acyl chains in MS/MS spectra from positive ion mode, giving information of fatty acid composition (Figure S1). Therefore, phospholipids were quantified using chromatographic areas in negative ion mode since they provide the acyl chain component information, whereas TG and DG were quantified in positive ion mode. The quantitation was adjusted using the models in Figure S2 to correct for the effect of ${ }^{13} \mathrm{C}$ heavy isotopes and response factors for various acyl chain length $[38,39]$.

3.2. Global Profiling of Phospholipids and Glycerolipids. In order to compare the strain or organ dependence of lipid constituents, phospholipids, and glycerolipids including LysoPC, LysoPE, LysoPS, PC, PE, PG, PS, PI, CL, DG, and TG in 12 organs/tissues from Wistar rats, CD1 and NMRI mice were profiled. There were 9 replicates for each organ/ tissue. A total of 1144 lipid molecules from 324 tissues were confidently identified based on MS/MS spectra match and quantified using chromatographic areas of precursors. The 


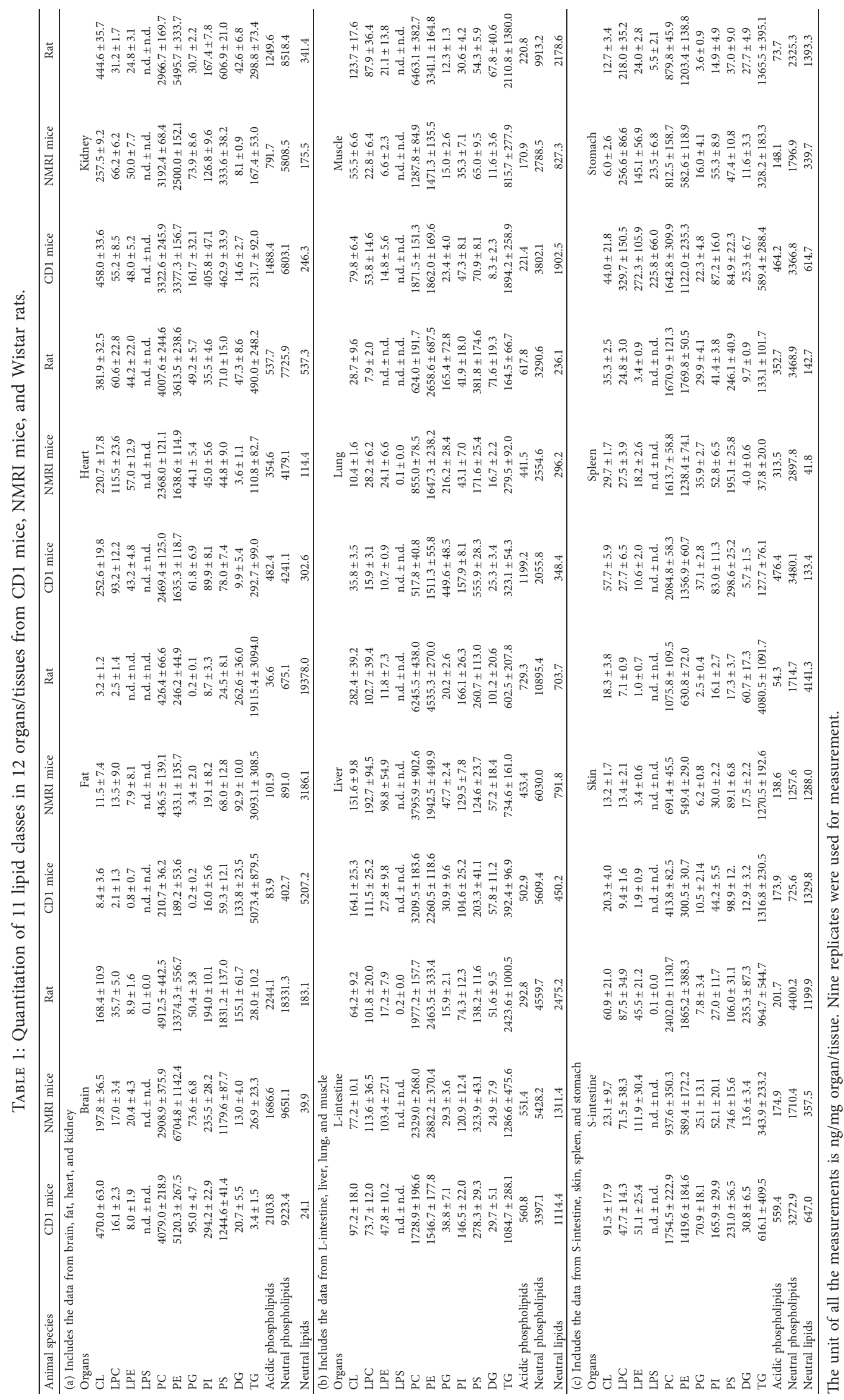


PE

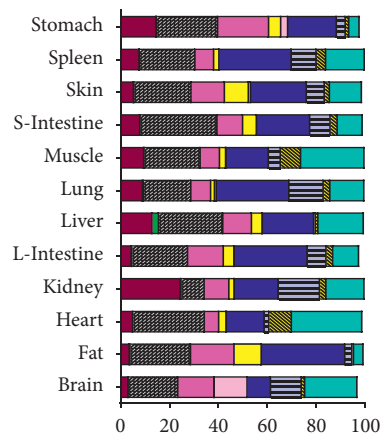

$\mathrm{PE}$

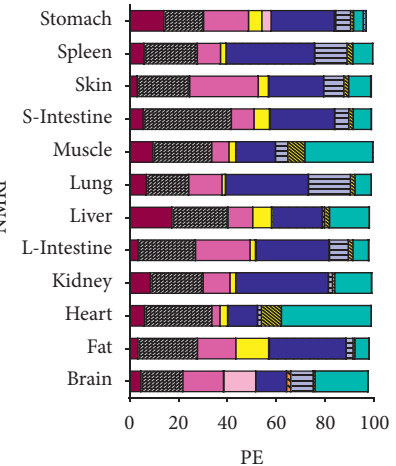

Stomach - 且目

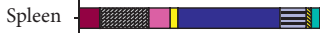

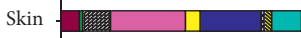

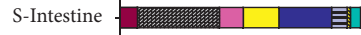

Muscle -

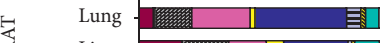

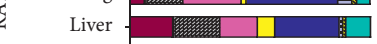

L-Intestine -

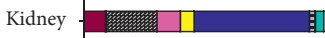

Heart -\begin{tabular}{r|r|}
-1 & $\mathbb{1}$ \\
\hline
\end{tabular}

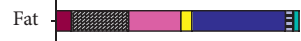

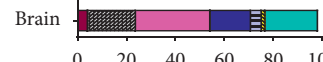
PS

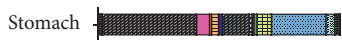

Spleen - खिए।

Skin $-1 \mid$ 름

S-Intestine -

Muscle

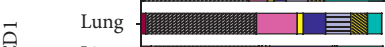

Liver - 25:

L-Intestine

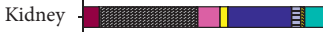

Heart 形㻣

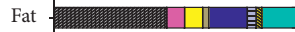

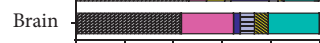

$\begin{array}{llllll}0 & 20 & 40 & 60 & 80 & 100\end{array}$
PC

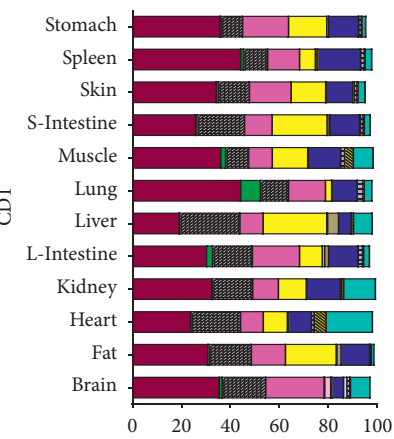

PC
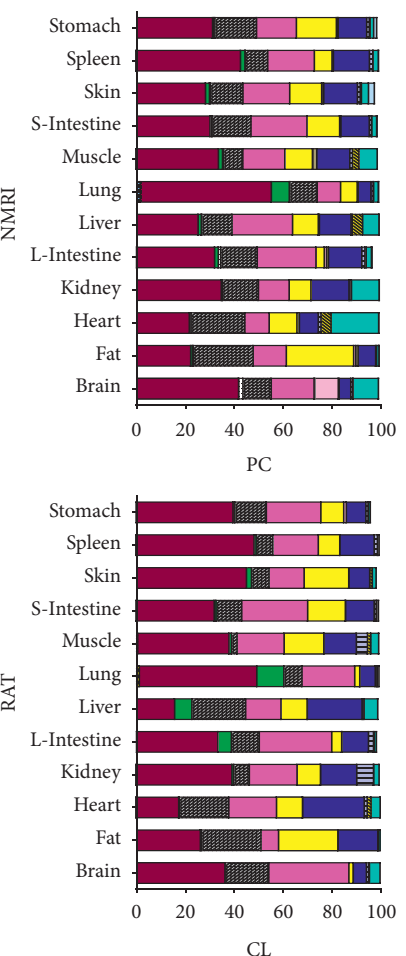

Stomach - D Spleen - III

Skin -

S-Intestine

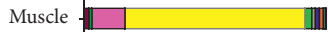

$\overrightarrow{0} \quad$ Lung

Liver $-\|$ I I I

L-Intestine $-\square \square$

Kidney

Heart $\square$

Fat -

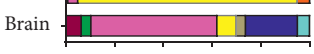

PI

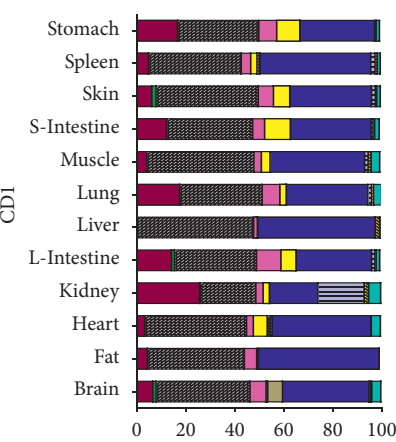

PI

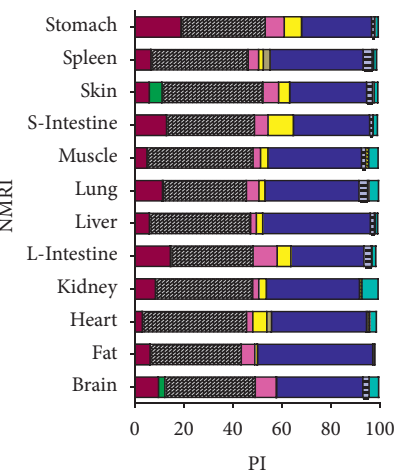

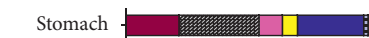

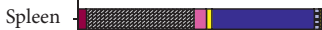

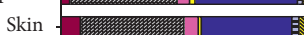

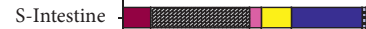

Muscle

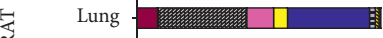

Liver - -

L-Intestine - |

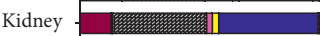

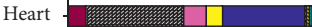

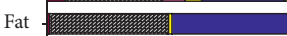

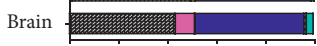
$\begin{array}{llllll}0 & 20 & 40 & 60 & 80 & 100\end{array}$ DG

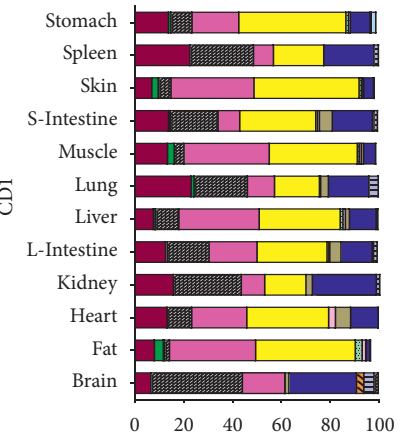

PG

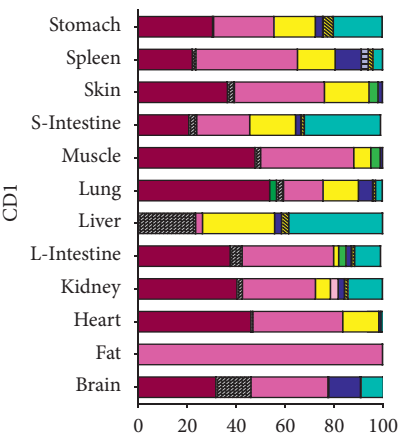

PG
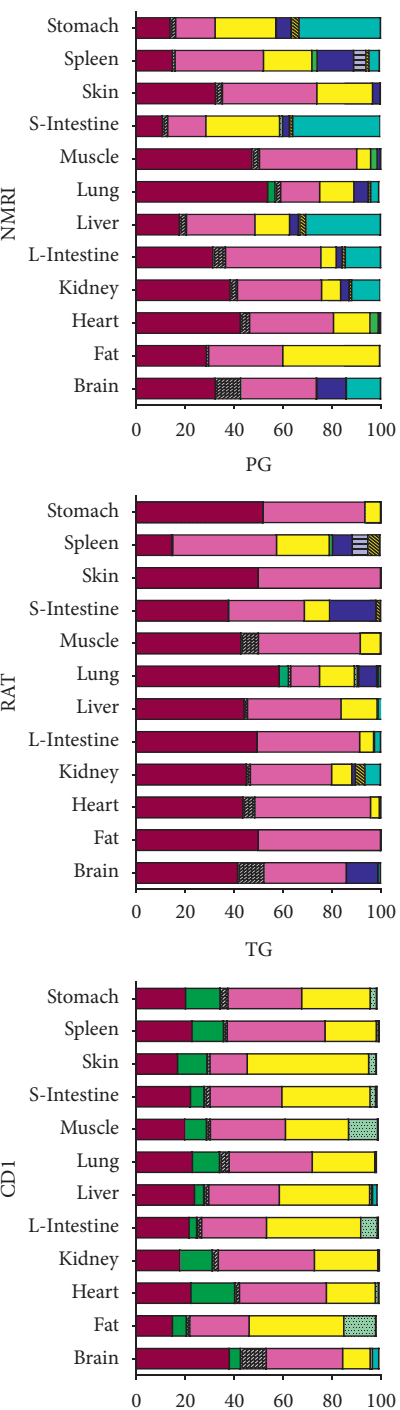

(a)

Figure 2: Continued. 

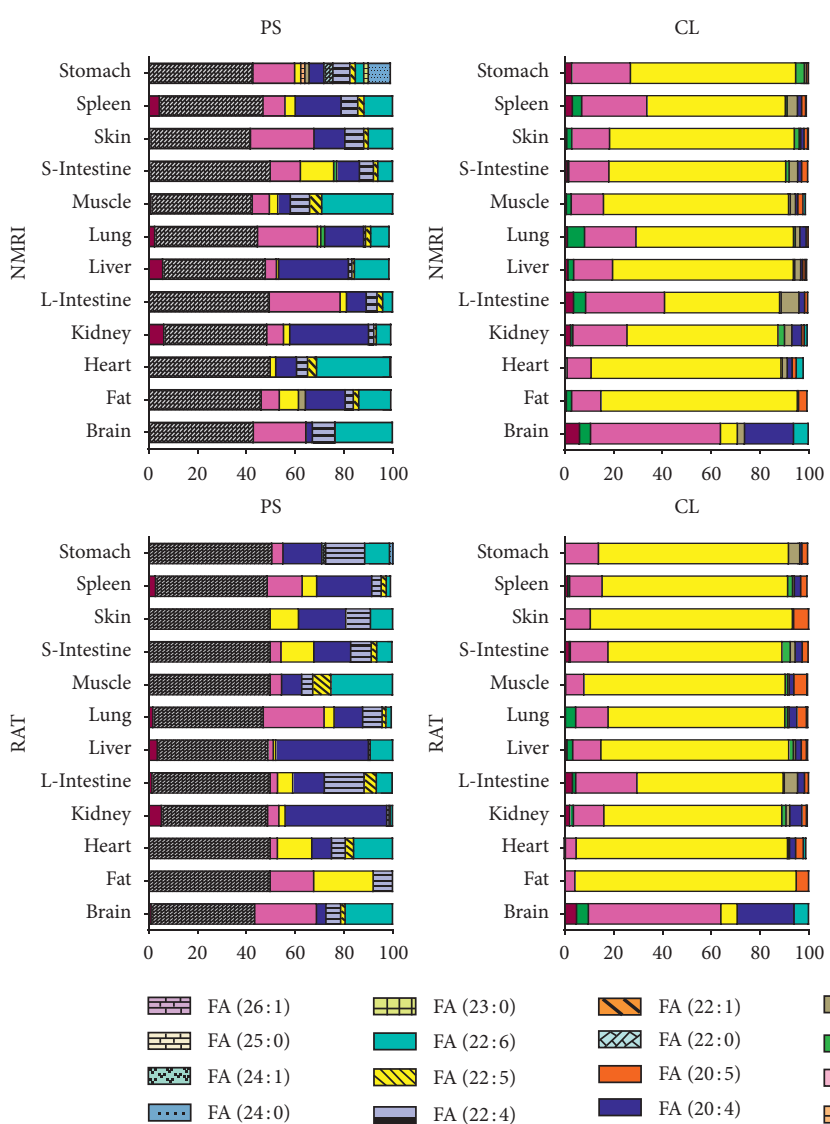
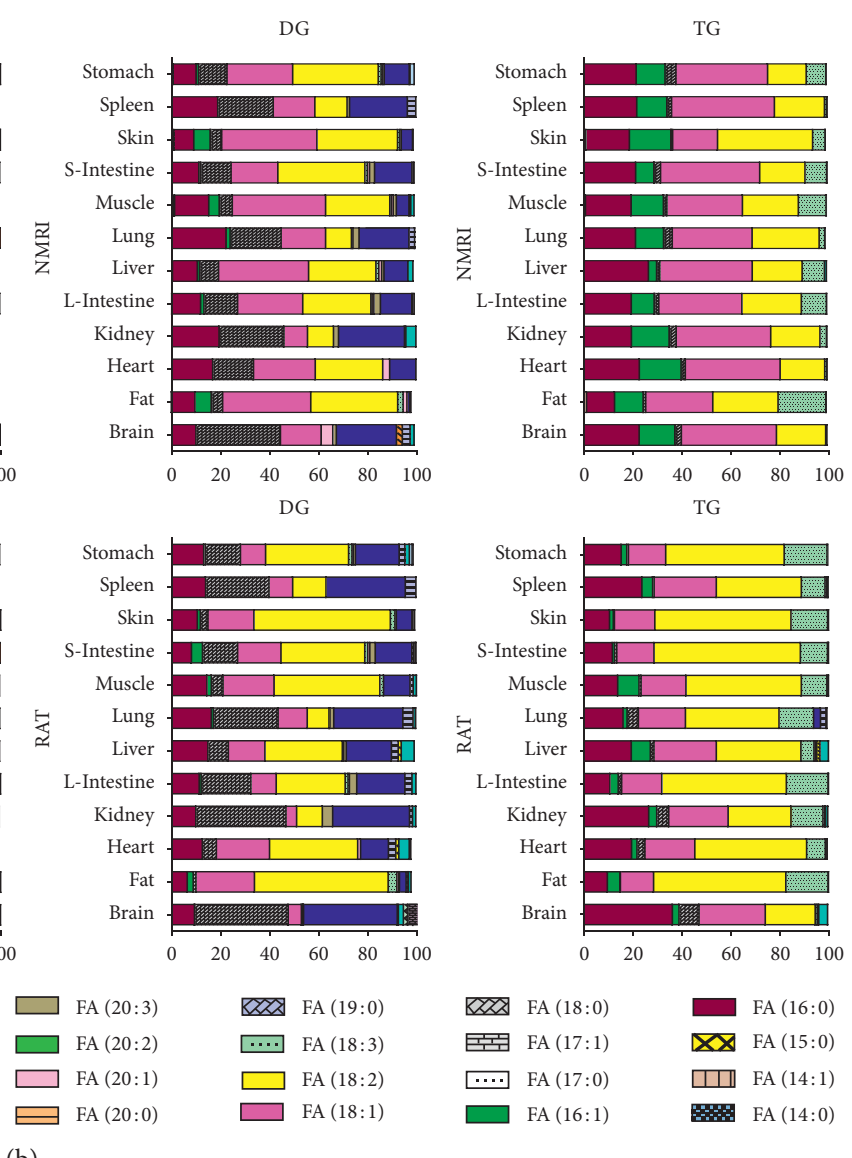

(b)

FIGURE 2: Acyl chain percentage compositions of PE, PC, PI, PG, PS, CL, DG, and TG in 12 organs/tissues of CD1, NMRI mice, and rats $(n=9)$. Each color represents a different fatty acid chain. The acyl chains with less than $1 \%$ were not included in the figures for better visualization.

quantitation of these 11 lipid classes in each organ/tissue is displayed in Table 1. Phosphatidic acid (PA) was excluded from the analysis due to the poor linearity and chromatographic behavior in this method. Overall, organ specificity was more obvious compared to animal species dependency. Lung has the highest PG level among all the organ/tissues, whereas brain tissues contain much higher levels of PS lipids in all animals. Notably, the most abundant lysophospholipid levels were observed in the stomach, especially for lysophosphatidylcholine. It has been known that lysophospholipids facilitate fat emulsion and nutrient absorption in the digestive system. Therefore, high levels of lysophospholipids in the stomach may be related to the unique function of these lipid molecules. In order to use the results as input into tissue composition-based equations of PBPK models [40], the lipids were classified as acidic phospholipids (CL, PG, PI, and PS), neutral phospholipids (LPC, PC, LPE, and PE), and neutral lipids (DG and TG) and the amounts were calculated by the sum of corresponding lipid levels as listed in Table 1. However, in our measurement, the brain has the most abundant acidic phospholipids in all three animal species due to its high level of PS.
3.3. Acyl Chain Components in Phospholipids and Glycerolipids. All phospholipids and glycerolipids were composed of acyl chains with diverse chain length and double bonds. It has been proposed that the acyl chain composition may play a biological function due to the diverse structures [41, 42]. In our method, the fatty acid compositions of phospholipids and glycerolipids in animal organs/tissues were able to be profiled and compared. Figure 2 demonstrates the acyl chain compositions of PC, PE, PS, PG, PI, CL, DG, and TG in 12 organs/tissues of CD1 and NMRI mice as well as Wistar rats. The acyl chains with less than $1 \%$ were not included in the figures for better visualization. Clearly, animal strain specificity was rarely observed among these three animals for most lipids. The heatmaps in Figure 3 confirmed the clustering of fatty acid constituents in 12 organs/tissues of three animal strains. More obvious animal strain similarity was observed based on the clustering results, in which muscle, skin, heart, liver, and fat tissues are more alike. Among all the organs/tissues, heart and liver are highly similar in fatty acid components.

However, a certain animal strain dependency of acyl chain components was seen for some lipid species. 

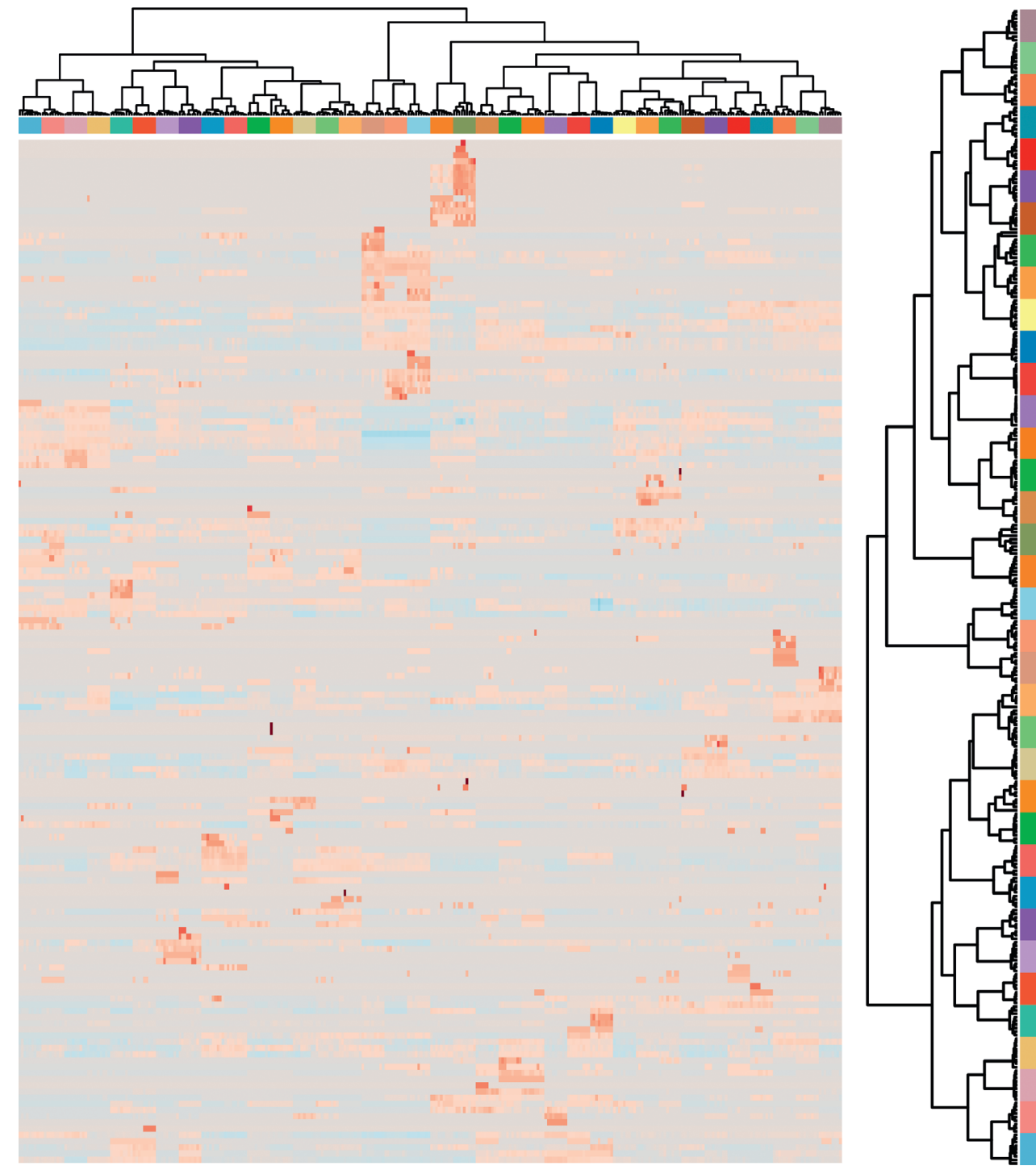

Rat lung

CD lung

NMRI lung

CD L-intestine

NMRI L-intestine

Rat L-intestine

Rat stomach

CD S-intestine

NMRI S-intestine

Rat S-intestine

CD kidney

NMRI kidney

Rat kidney

NMRI spleen

CD spleen

Rat spleen

CD stomach

NMRI stomach

$\mathrm{CD}$ brain

Rat brain

NMRI brain

NMRI muscle

CD muscle

Rat muscle

NMRI skin

CD skin

NMRI heart

CD heart

Rat liver

Rat heart

NMRI liver

CD liver

Rat skin

Rat fat

NMRI fat

$\mathrm{CD}$ fat

FIGURE 3: Heatmap clustering of fatty acid constituents in 12 organs/tissues of CD1 and NMRI mice and rats $(n=9)$. Analysis was performed using MetaboAnalyst (http://www.metaboanalyst.ca). Distance measure of Elucidation and clustering algorithm of Ward were applied in the analysis.

Phosphatidylcholine (PC) and phosphatidylglycerol (PG) tended to incorporate more FA $(22: 6)$ in CD1 and NMRI mice compared to Wistar rats, especially for brain, heart, small intestine, stomach, and liver (Figures 4(a) and 4(b)). In contrast, triglycerides in Wistar rats had more preference of FA $(18: 3)$ compared to CD1 and NMRI mice (Figure 4(c)). In Figure 2, the similarity of acyl chain constituents among different organs/tissues was observed with the exception of the brain. FA $(18: 2)$ is extremely unfavored in brain tissue for all phospholipids and diglycerides. Although FA (18:2) accounts for about $70 \%$ of the cardiolipin acyl components in all other tissues, the brain only contains $\sim 7 \%$ of FA $(18: 2)$ and favors FA (18:1) and FA $(20: 4)$ instead (Figure 4(d)).

Lipid remodeling is a well-known strategy to help the lipids adapt a specific biological function in various organs/ tissues. Unlike the symmetric structure of cardiolipin with dominant FA (18:2) in mouse heart, liver, and muscle, a more diverse acyl chain composition has been reported in brain tissue $[43,44]$. Our profiling results demonstrate consistent observation and conclude that the brain is the only exceptional organ among all 12 organs/tissues. It has been hypothesized that the diversified fatty acid composition in cardiolipins is related to the reduced mitochondrial bioenergetic efficiency in the brain $[43,45]$. However, the overall unfavored incorporation of FA $(18: 2)$ was observed in most lipids except triglycerides (Figure 2). Free fatty acids were analyzed in all tissues/organs of the rodent species/strains tested. A relative abundance of free FA $(18: 2)$ in brain tissues was lower by at least an order of magnitude compared to all other tissues (Figure S3). Therefore, the composition of free fatty acids in the brain may be the result of unfavored incorporation of FA $(18: 2)$ in phospholipids and glycerolipids after lipid remodeling. Notably, the acyl chain components in triglycerides are distinct from the ones in diglycerides due to 


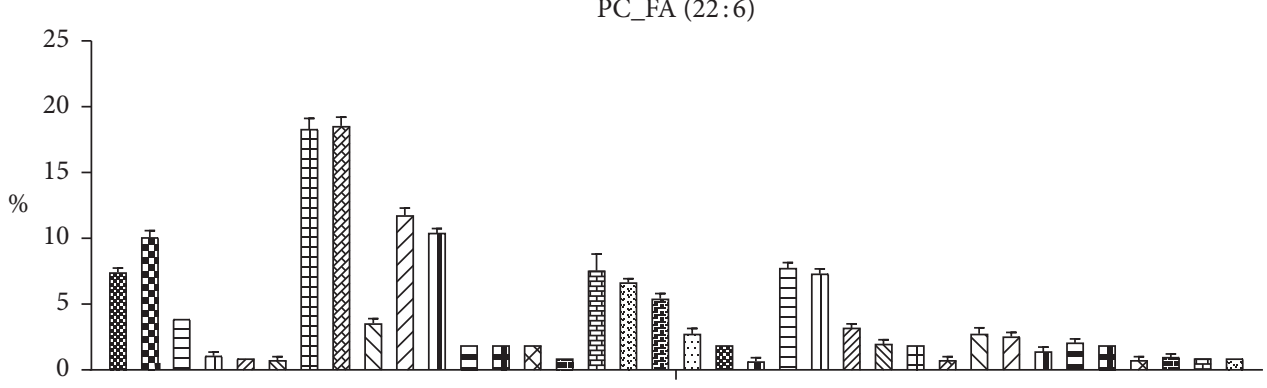

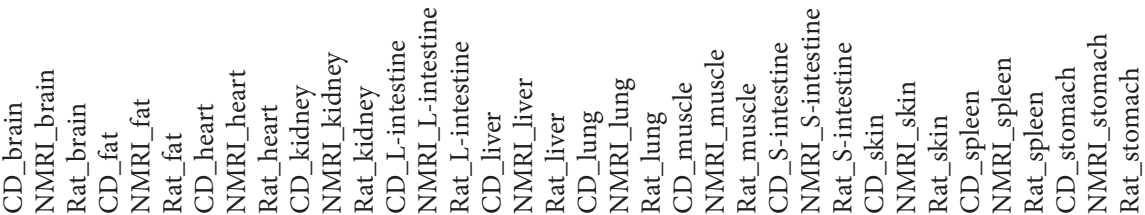

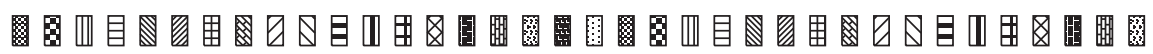

(a)

PG_FA $(22: 6)$

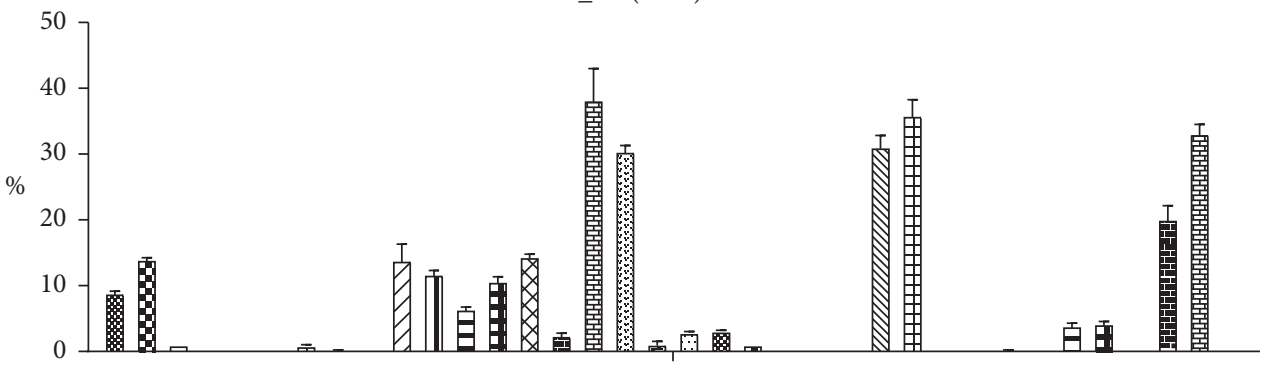

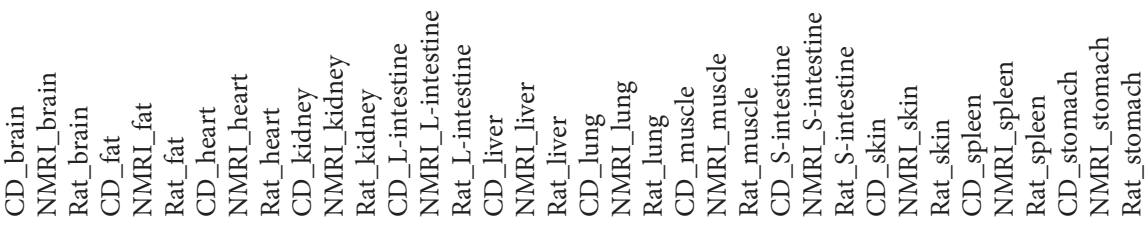

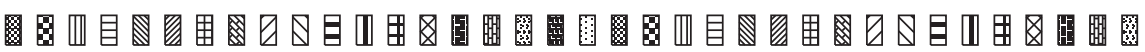

(b)

TG_FA (18:3)

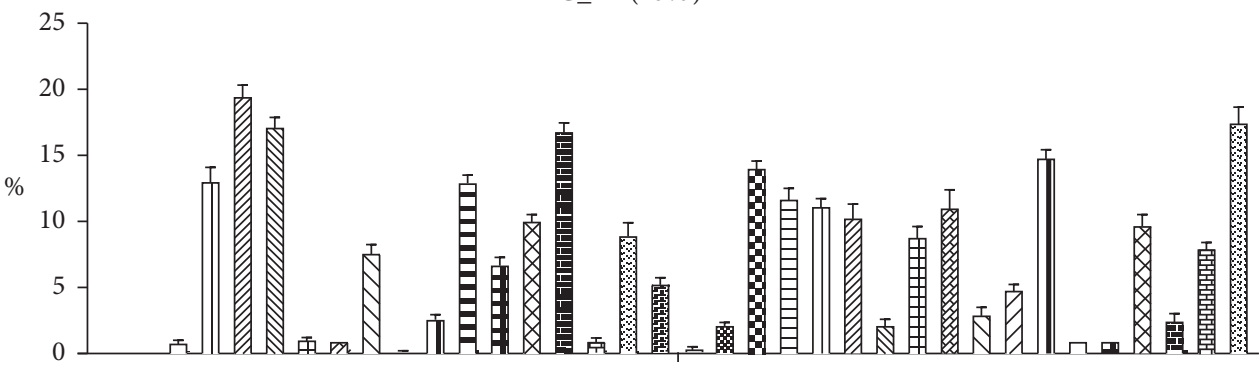

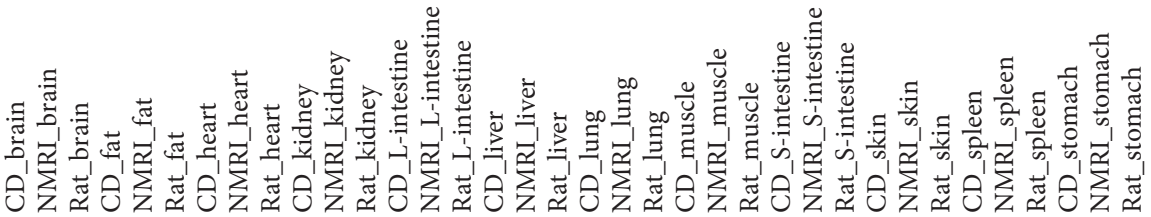

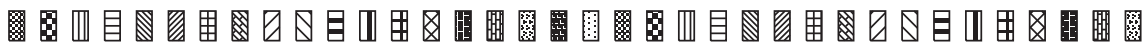

(c)

Figure 4: Continued. 

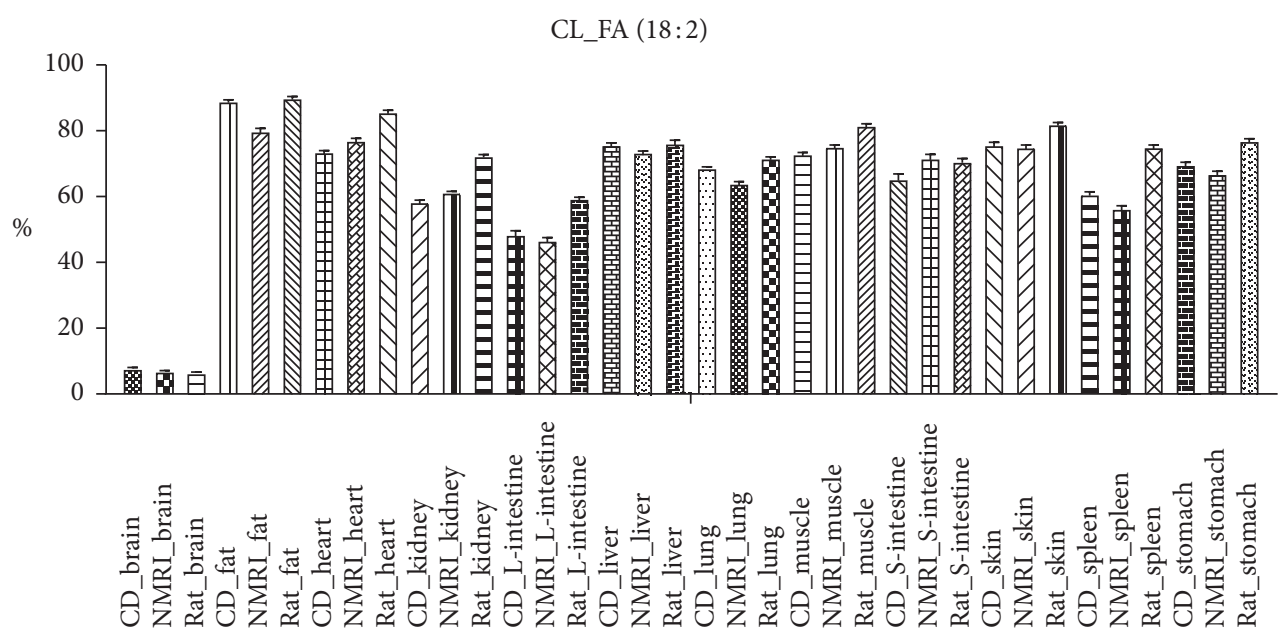

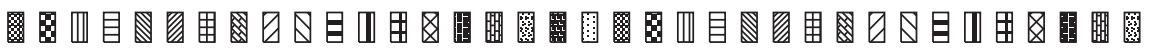

(d)

Figure 4: Percentage contribution of (a) acyl chain FA(22:6) in PC, (b) acyl chain FA(22:6) in PG, (c) acyl chain FA(18:3) in TG, and (d) acyl chain $\mathrm{FA}(18: 2)$ in CL in 12 organs/tissues of CD1 and NMRI mice and rats $(n=9)$.

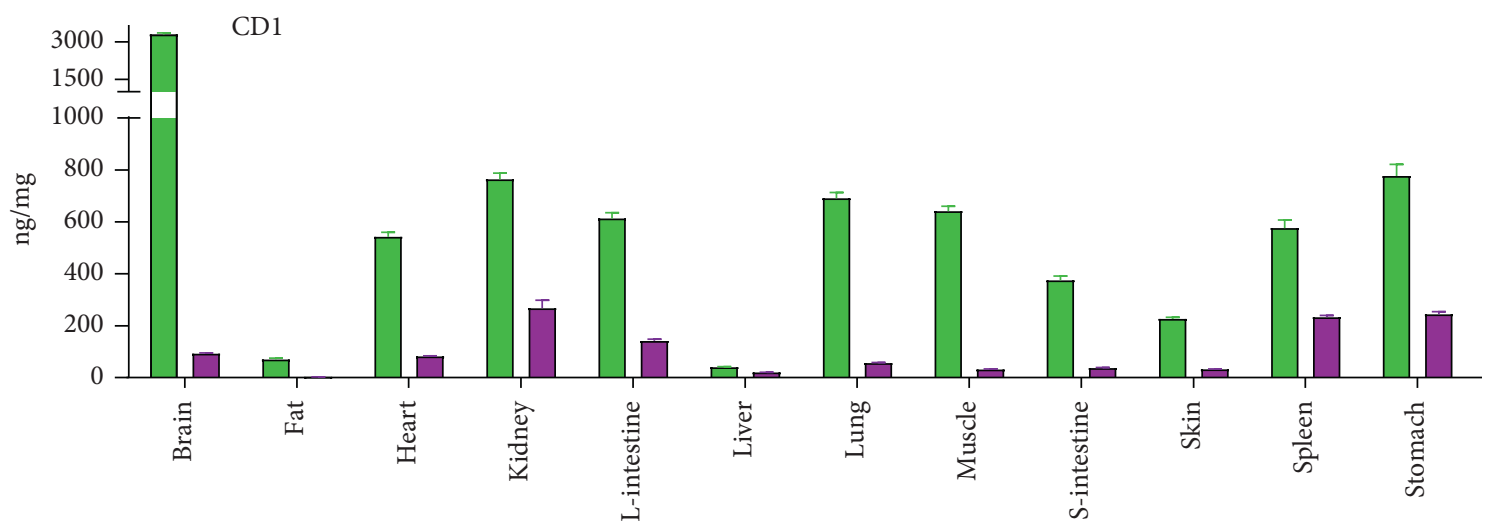

$\square$ ePE

(a)

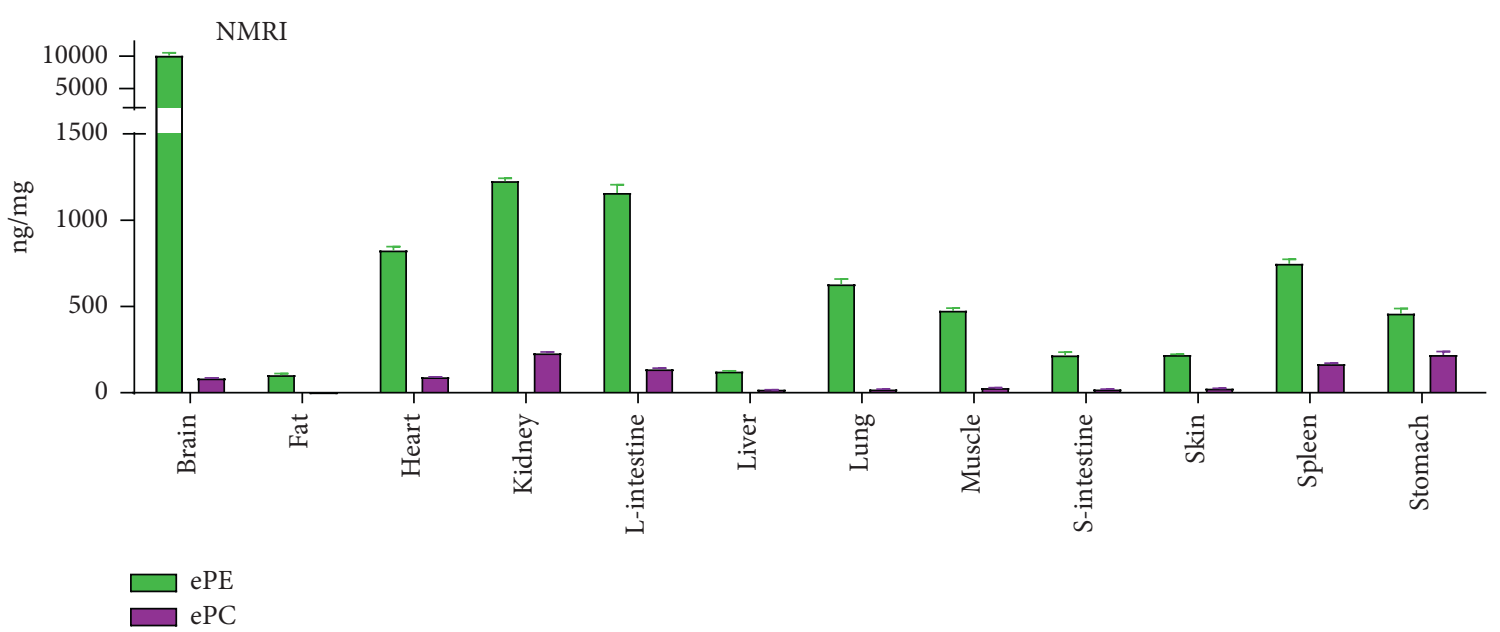

(b)

FIgURE 5: Continued. 


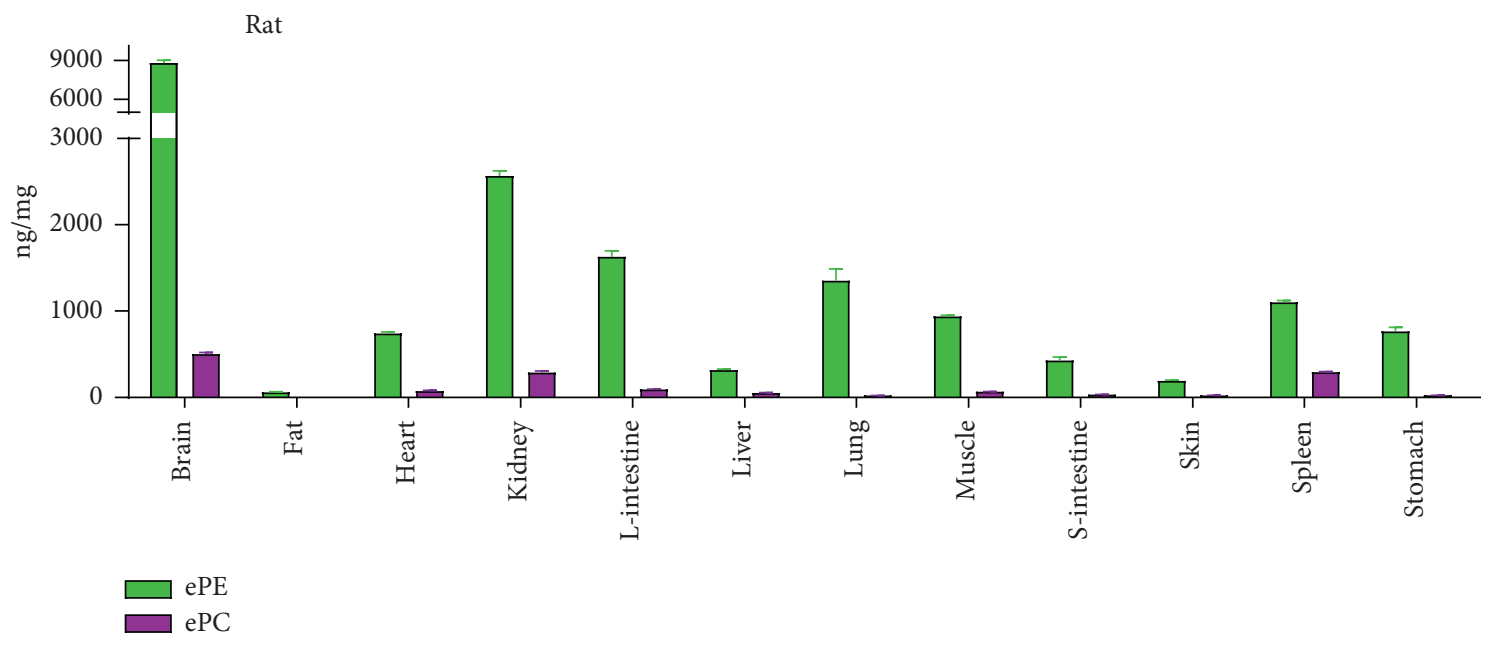

(c)

Figure 5: Absolute amounts of ether lipids as quantified in 12 organs/tissues from CD1 and NMRI mice and rats $(n=9)$.

the lipid remodeling process [46]. FA $(18: 0)$ and FA $(20: 4)$ contents were highly reduced in triglycerides, indicating scarce usage of these two fatty acids as energy store.

\subsection{Analysis of Ether Lipids in 12 Organs/Tissues of Rats and} Mice. Ether lipids refer to the phospholipid molecules with an alkyl or alkenyl chain attached to an ether bond instead of an acyl chain at sn-1 position. Two types of ether lipids, plasmanyl- and plasmenyl-phospholipids, exist in mammalian cells and animals $[47,48]$. Plasmanyl-phospholipids carry the ether bond at an alkyl group, whereas plasmenylphospholipids contain the ether bond at an alkenyl group at sn-1 position. Plasmenyl-phospholipids, normally called plasmalogens, can protect from cellular damage due to reactive oxygen species (ROS) and play a role in cell signaling processes $[5,49]$. The most common ether lipids are plasmanyl- and plasmenyl-phosphatidylcholines (referred to as ePC) and phosphatidylethanolamines (referred to as ePE).

The amounts of ether lipids were quantified in 12 organs/ tissues from Wistar rats and CD1 and NMRI mice as displayed in Figure 5. Although organ/tissue specificity was observed, high similarity within animal strains was found for the distribution of ether lipids in general. Apparently, among all the organs/tissues, brain contains the highest levels of ePEs. Only weak species differences were observed for ether lipid distribution among 12 organs/tissues, with CD1 mice containing much fewer ether lipids compared to NMRI mice and Wistar rats.

3.5. Analysis of Acyl Chain Components in Brain Tissue of Aged Mice. Using the method established above, the acyl chain constituents of phospholipids and glycerolipids in brain tissues of aged mice are analyzed in Figure S4. The percentage of acyl chain composition was calculated based on each lipid species. The results indicated that FA $(16: 1)$ in PI, FA $(17: 0)$ in PG, and FA $(16: 1 \mathrm{e})$ in PE had less percentage contribution in mice brain of 2 years old. However, FA (18: 2e) in PE, FA (22:6) in PG, and FA (19:0) in TG had higher incorporation level in lipids in aged mice brain.

\section{Conclusion}

In this work, we profiled the phospholipids and glycerolipids in 12 organs/tissues from Wistar rat and CD1 and NMRI mice. The high resolution MS/MS fragments were applied to assign the fatty acid component of lipid molecule and strategies to reduce false positive identifications were used to eliminate unreliable results. A total of 1144 lipids belonging to LysoPC, LysoPE, LysoPS, PC, PE, PG, PS, PI, CL, DG, and TG class were reliably identified and relatively quantified. Organ dependency of phospholipids and glycerolipids was observed in all three animal species/stains examined. The acyl chain components in 12 organs/tissues of mice and rats also revealed that each lipid class has a unique pattern of fatty acid compositions. However, rare animal species specificity was observed except for FA $(22: 6)$ in PC and PG. An unfavored FA $(18: 2)$ incorporation in phospholipids, especially in cardiolipins was observed in brain tissue. The analysis of free fatty acids turned out that the deficiency of FA $(18: 2)$ in brain tissues may be caused by the access of this FF. The plasmenyl-phosphatidylethanolamines (ePEs) were the most abundant ether lipids in all organs/tissues, with the brain containing the highest levels of ePEs. This supports the potential function of ether lipids, especially plasmalogens in neurodegenerative diseases. The aged mice were applied using this approach to investigate the change of fatty acid compositions during the aging process. We did observe certain acyl chain in PG, PI, and TG had changed percentage contributions in 2-year-old mouse brains. In PE lipids, only ether chain showed the difference during aging. Overall, the systematic analysis of the lipid molecule distribution in all major organs/tissues of Wistar rats, NMRI mice, and CD1 mice provides a coherent basis for the prediction of drug partition coefficient using mechanistic tissue compositionbased equations commonly used in PBPK modeling [50]. 


\section{Data Availability}

The data that are used to support the findings of this study are available from the corresponding author upon request.

\section{Conflicts of Interest}

The authors declare no conflicts of interest.

\section{Acknowledgments}

The authors would like to thank Petra Kölsch, Frank Fiske, Maria Gülden-Steinke, Anja Duschka, and Juliane Trieloff for tissue generation, as well as Yanhua Tian and Ming Niu for sample preparation and data analysis. The authors also thank Dr. Yu Xia, Dr. Xianlin Han, and Dr. Haiteng Deng for advice. The authors are grateful for the help of Metabolomics and Lipidomics Center at Tsinghua University. The work was funded by a research grant from Bayer AG (20153000383) and National Natural Science Foundation of China (81501776 and 81871093).

\section{Supplementary Materials}

Figure S1: Features MS/MS spectra of PC, PE, PG, PI, PS, and $C L$ in negative ion mode and DG, TG in positive ion mode, respectively. Figure S2: (a) Equation to correct the effect of natural 13C heavy isotopes for quantitation of glycerophospholipids and glycerolipids. (b) Equation to correct the response factors of various acyl chain length based on references for quantitation of glycerolipids. Figure S3: Abundance percentage of free $\mathrm{FA}(18: 2)$ in all detected free fatty acids in 12 organs/tissues in (a) CD1 mice, (b) NMRI mice, and (c) Wistar rats. Figure S4: Heatmap of acyl chain constituents of glycerophospholipids and glycerolipids in brain tissues of mice at the age of 12 weeks and 96 weeks. Analysis was performed using MetaboAnalyst (). Distance measure of Elucidation and clustering algorithm of Ward were applied in the analysis. (Supplementary Materials)

\section{References}

[1] M. C. F. Messias, G. C. Mecatti, D. G. Priolli, and P. de Oliveira Carvalho, "Plasmalogen lipids: functional mechanism and their involvement in gastrointestinal cancer," Lipids in Health and Disease, vol. 17, no. 4, p. 41, 2018.

[2] I. Lamiquiz-Moneo, F. Civeira, D. Gomez-Coronado et al., "Lipid profile rather than the LCAT mutation explains renal disease in familial LCAT deficiency," Journal of Clinical Medicine, vol. 8, no. 11, p. 1860, 2019.

[3] V. Villafuerte-Ledesma and D. B. Goodenowe, "Plasmalogen deficiency and neuropathology in Alzheimer's disease: causation or coincidence?" Alzheimer's \& Dementia: Translational Research \& Clinical Interventions, vol. 5, no. 1, pp. 524-532, 2019.

[4] H. Xicoy, B. Wieringa, and G. J. M. Martens, "The role of lipids in Parkinson's disease," Cells, vol. 8, no. 1, p. 7, 2019.

[5] N. E. Braverman and A. B. Moser, "Functions of plasmalogen lipids in health and disease," Biochimica et Biophysica Acta, vol. 1822, no. 9, pp. 1442-1452, 2012.
[6] M. Mapstone, A. K. Cheema, M. S. Fiandaca et al., "Plasma phospholipids identify antecedent memory impairment in older adults," Nature Medicine, vol. 20, no. 4, pp. 415-418, 2014.

[7] R. Laaksonen, K. Ekroos, M. Sysi-Aho et al., "Plasma ceramides predict cardiovascular death in patients with stable coronary artery disease and acute coronary syndromes beyond LDL-cholesterol," European Heart Journal, vol. 37, no. 25, pp. 1967-1976, 2016.

[8] G. Lüscher, L. Wang, P. C. Marcogliese, and H. J. Bellen, "Sphingolipids in the pathogenesis of Parkinson's disease and parkinsonism," Trends in Endocrinology \& Metabolism, vol. 30, no. 2, pp. 106-117, 2019.

[9] Y. A. Hannun and L. M. Obeid, "Sphingolipids and their metabolism in physiology and disease," Nature Reviews Molecular Cell Biology, vol. 19, no. 3, pp. 175-191, 2018.

[10] P. J. Meikle and S. A. Summers, "Sphingolipids and phospholipids in insulin resistance and related metabolic disorders," Nature Reviews Endocrinology, vol. 13, no. 2, pp. 79-91, 2017.

[11] N. Yata, T. Toyoda, T. Murakami, A. Nishiura, and Y. Higashi, "Phosphatidylserine as a determinant for the tissue distribution of weakly basic drugs in rats," Pharmaceutical Research, vol. 07, no. 10, pp. 1019-1025, 1990.

[12] T. Rodgers, D. Leahy, and M. Rowland, "Physiologically based pharmacokinetic modeling 1: predicting the tissue distribution of moderate-to-strong bases," Journal of Pharmaceutical Sciences, vol. 94, no. 6, pp. 1259-1276, 2005.

[13] P. Poulin, Y.-H. Chen, X. Ding et al., "Prediction of drug distribution in subcutaneous xenografts of human tumor cell lines and healthy tissues in mouse: application of the tissue composition-based model to antineoplastic drugs," Journal of Pharmaceutical Sciences, vol. 104, no. 4, pp. 1508-1521, 2015.

[14] P. Hop, "Drug distribution to human tissues: prediction and examination of the basic assumption in in vivo pharmacokinetics-pharmacodynamics (PK/PD) research," Journal of Pharmaceutical Sciences, vol. 104, no. 6, pp. 2110-2118, 2015.

[15] P. Poulin and F. P. Theil, "A priori prediction of tissue:plasma partition coefficients of drugs to facilitate the use of physiologically-based pharmacokinetic models in drug discovery," Journal of Pharmaceutical Sciences, vol. 89, no. 1, pp. 16-35, 2000.

[16] T. Rodgers, H. M. Jones, and M. Rowland, “Tissue lipids and drug distribution: dog versus rat," Journal of Pharmaceutical Sciences, vol. 101, no. 12, pp. 4615-4626, 2012.

[17] W. Escorcia, D. L. Ruter, J. Nhan, and S. P. Curran, "Quantification of lipid abundance and evaluation of lipid distribution in Caenorhabditis elegans by nile red and oil red O staining," Journal of Visualized Experiments Jove, vol. 133, p. 57352, 2018.

[18] S. Naoe, H. Tsugawa, M. Takahashi, K. Ikeda, and M. Arita, "Characterization of lipid profiles after dietary intake of polyunsaturated fatty acids using integrated untargeted and targeted lipidomics," Metabolites, vol. 9, no. 10, p. 241, 2019.

[19] J. Bredehöft, D. R. Bhandari, F. J. Pflieger et al., "Visualizing and profiling lipids in the OVLT of fat-1 and wild type mouse brains during LPS-induced systemic inflammation using APSMALDI MSI," ACS Chemical Neuroscience, vol. 10, no. 10, pp. 4394-4406, 2019.

[20] R. W. Kang, "The evolution of lipidomics through space and time," Biochimica et Biophysica Acta (BBA)-Molecular and Cell Biology of Lipids, vol. 1862, no. 8, pp. 731-739, 2017. 
[21] M. Wang, C. Wang, R. H. Han, and X. Han, "Novel advances in shotgun lipidomics for biology and medicine," Progress in Lipid Research, vol. 61, pp. 83-108, 2016.

[22] J. Wang, C. Wang, and X. Han, "Tutorial on lipidomics," Analytica Chimica Acta, vol. 1061, pp. 28-41, 2019.

[23] L. Lu, C. Hu, Y. Fan et al., "Shotgun lipidomics revealed altered profiles of serum lipids in systemic lupus erythematosus closely associated with disease activity," Biomolecules, vol. 8, no. 4, pp. 28-41, 2018.

[24] J. Chen and K. K. Nichols, "Comprehensive shotgun lipidomics of human meibomian gland secretions using MS/ MSallwith successive switching between acquisition polarity modes," Journal of Lipid Research, vol. 59, no. 11, pp. 2223-2236, 2018.

[25] H.-C. Lee and T. Yokomizo, "Applications of mass spectrometry-based targeted and non-targeted lipidomics," Biochemical and Biophysical Research Communications, vol. 504, no. 3, pp. 576-581, 2018.

[26] S. M. Lam, H. Tian, and G. Shui, "Lipidomics, en route to accurate quantitation," Biochimica et Biophysica Acta (BBA)Molecular and Cell Biology of Lipids, vol. 1862, no. 8, pp. 752-761, 2017.

[27] Q. Zhang, H. Xu, R. Liu et al., "A novel strategy for targeted lipidomics based on LC-tandem-MS parameters prediction, quantification, and multiple statistical data mining: evaluation of lysophosphatidylcholines as potential cancer biomarkers," Analytical Chemistry, vol. 91, no. 5, pp. 3389-3396, 2019.

[28] E. L. Yang, J. P. Koelmel, L. Meke et al., "Ultrahigh-Performance liquid chromatography-high-resolution mass spectrometry metabolomics and lipidomics study of stool from transgenic Parkinson's disease mice following immunotherapy," Journal of Proteome Research, vol. 19, no. 1, pp. 424-431, 2019.

[29] A. M. Yost, R. D. Trengove, I. D. Wilson et al., "Rapid profiling method for the analysis of lipids in human plasma using ion mobility enabled-reversed phase-ultra high performance liquid chromatography/mass spectrometry," Journal of Chromatography A, vol. 1611, p. 460597, 2019.

[30] T. S. Anthonymuthu, E. M. Kenny, A. M. Lamade et al., "Lipidomics detection of brain cardiolipins in plasma is associated with outcome after cardiac arrest," Critical Care Medicine, vol. 47, no. 4, pp. e292-e300, 2019.

[31] S. Krehel, A. Criscuolo, M. Zeller, M. Fedorova, M. R. Domingues, and P. Domingues, "Analysis of oxidised and glycated aminophospholipids: complete structural characterisation by C30 liquid chromatography-high resolution tandem mass spectrometry," Free Radical Biology and Medicine, vol. 144, pp. 144-155, 2019.

[32] T. Kind, K.-H. Liu, D. Y. Lee, B. DeFelice, J. K. Meissen, and O. Fiehn, "LipidBlast in silico tandem mass spectrometry database for lipid identification," Nature Methods, vol. 10, no. 8, pp. 755-758, 2013.

[33] E. Fahy, M. Sud, D. Cotter, and S. Subramaniam, "LIPID MAPS online tools for lipid research," Nucleic Acids Research, vol. 35, no. Web Server, pp. W606-W612, 2007.

[34] M. Narváez-Rivas and Q. Zhang, "Comprehensive untargeted lipidomic analysis using core-shell C30 particle column and high field orbitrap mass spectrometer," Journal of Chromatography A, vol. 1440, pp. 123-134, 2016.

[35] Z. Zhou, J. Tu, and Z.-J. Zhu, "Advancing the large-scale CCS database for metabolomics and lipidomics at the machinelearning era," Current Opinion in Chemical Biology, vol. 42, pp. 34-41, 2018.
[36] X. Wang, M. V. Schmitt, L. Xu et al., "Quantitative molecular tissue atlas of Bis(monoacylglycero) phosphate and phosphatidylglycerol membrane lipids in rodent organs generated by methylation assisted high resolution mass spectrometry," Analytica Chimica Acta, vol. 1084, pp. 60-70, 2019.

[37] L. Guo, X. Wang, Y. Jiao, and X. Liu, "Assessment of potential false positives via orbitrap-based untargeted lipidomics from rat tissues," Talanta, vol. 178, pp. 287-293, 2018.

[38] X. Han and R. W. Gross, "Quantitative analysis and molecular species fingerprinting of triacylglyceride molecular species directly from lipid extracts of biological samples by electrospray ionization tandem mass spectrometry," Analytical Biochemistry, vol. 295, no. 1, pp. 88-100, 2001.

[39] M. Wang, C. Wang, and X. Han, "Selection of internal standards for accurate quantification of complex lipid species in biological extracts by electrospray ionization mass spectrometry-what, how and why?" Mass Spectrometry Reviews, vol. 36, no. 6, pp. 693-714, 2017.

[40] T. Peyret, P. Poulin, and K. Krishnan, "A unified algorithm for predicting partition coefficients for PBPK modeling of drugs and environmental chemicals," Toxicology and Applied Pharmacology, vol. 249, no. 3, pp. 197-207, 2010.

[41] K. D'Souza and R. M. Epand, "Enrichment of phosphatidylinositols with specific acyl chains," Biochimica et Biophysica Acta (BBA)-Biomembranes, vol. 1838, no. 6, pp. 1501-1508, 2014.

[42] R. M. Epand, "Recognition of polyunsaturated acyl chains by enzymes acting on membrane lipids," Biochimica et biophysica acta, vol. 1818, no. 4, pp. 957-962, 2012.

[43] H. Cheng, D. J. Mancuso, X. Jiang et al., "Shotgun lipidomics reveals the temporally dependent, highly diversified cardiolipin profile in the mammalian brain: temporally coordinated postnatal diversification of cardiolipin molecular species with neuronal remodeling $\dagger$," Biochemistry, vol. 47, no. 21, pp. 5869-5880, 2008.

[44] X. Yang, K. Yang, J. Yang, H. Cheng, and R. W. Gross, "Shotgun lipidomics of cardiolipin molecular species in lipid extracts of biological samples," Journal of Lipid Research, vol. 47, no. 4, pp. 864-879, 2006.

[45] A. J. Chicco and G. C. Sparagna, "Role of cardiolipin alterations in mitochondrial dysfunction and disease," American Journal of Physiology-Cell Physiology, vol. 292, no. 1, pp. C33-C44, 2007.

[46] R. H. Han, M. Wang, X. Fang, and X. Han, "Simulation of triacylglycerol ion profiles: bioinformatics for interpretation of triacylglycerol biosynthesis," Journal of Lipid Research, vol. 54, no. 4, pp. 1023-1032, 2013.

[47] J. M. Dean and I. J. Lodhi, "Structural and functional roles of ether lipids," Protein \& Cell, vol. 9, no. 2, pp. 196-206, 2018.

[48] N. Jimenez-Rojo and H. Riezman, "On the road to unraveling the molecular functions of ether lipids," FEBS Letters, vol. 593, no. 17, pp. 2378-2389, 2019.

[49] R. A. Zoeller, A. C. Lake, N. Nagan, D. P. Gaposchkin, M. A. Legner, and W. Lieberthal, "Plasmalogens as endogenous antioxidants: somatic cell mutants reveal the importance of the vinyl ether," The Biochemical Journal, vol. 338, no. Pt 3, pp. 769-776, 1999.

[50] P. Poulin and S. Haddad, "Advancing prediction of tissue distribution and volume of distribution of highly lipophilic compounds from a simplified tissue-composition-based model as a mechanistic animal alternative method," Journal of Pharmaceutical Sciences, vol. 101, no. 6, pp. 2250-2261, 2012. 